$N=20$

NASA Technical Memorandum 106812

AIAA-95-0030

P. 11

\title{
A Modular Electric Power System Test Bed for Small Spacecraft
}

Robert M. Button and Anastacio N. Baez

Lewis Research Center

Cleveland, Ohio

Prepared for the

33rd Aerospace Sciences Meeting and Exhibit

sponsored by the American Institute of Aeronautics and Astronautics

Reno, Nevada, January 9-12, 1995
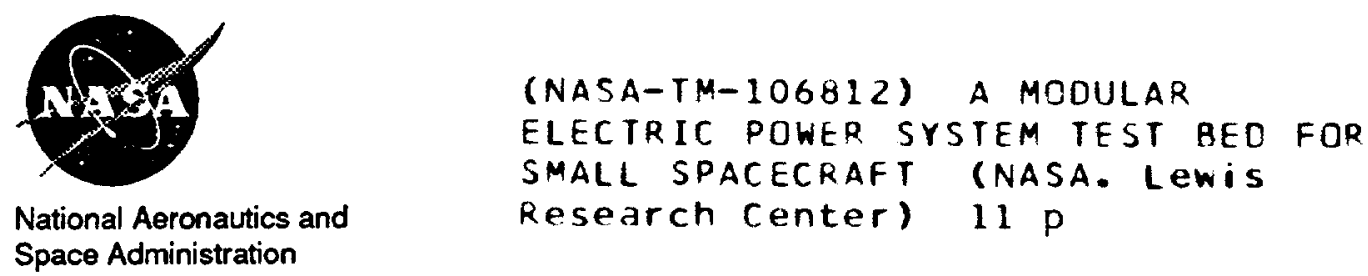
,$\ldots$

' 


\title{
A MODULAR ELECTRIC POWER SYSTEM TEST BED FOR SMALL SPACECRAFT
}

\author{
Robert M. Button \\ Anastacio N. Baez \\ National Aeronautics and Space Administration \\ Lewis Research Center \\ Cleveland, $\mathrm{OH} \mathbf{4 4 1 3 5}$
}

\begin{abstract}
ABSTRACI
In the new climate of smaller, faster, and cheaper space science satellites, a new power system topology has been developed at the NASA Lewis Research Center. This new topology is based on a series connected boost converter (SCBC) and can greatly affect the size, weight, fault tolerance, and cost of any small spacecraft using photovoltaic solar arrays. The paper presents electric power system design factors and requirements as background information. The series connected boost converter topology is discussed and several advantages over existing technologies are illustrated. Besides being small, lightweight, and efficient, this topology has the added benefit of inherent fault tolerance. A positive ground power system test bed has been developed for the TROPIX spacecraft program. Performance of the SCBC in the test bed is described in detail. SCBC efficiencies of $95 \%$ to $98 \%$ have been measured. Finally, a modular, photovoltaic regulator "kit" concept is presented. Two (2) SCBCs are used to regulate solar array charging of batteries and to provide "utility-type" power to the user loads. The kit's modularity will allow a spacecraft electric power system to be built from off-the-shelf hardware; resulting in smaller, faster, and cheaper spacecraft.
\end{abstract}

\section{INTRODUCTION}

The recent failures of several large spacecraft have led NASA to rethink its policy towards space science missions. The initial problems with the Hubble Space Telescope and the loss of the Mars Observer have drawn much public attention-their big price tags and long development times have put them under the scrutiny of Congress and the public. Although space science is difficult and risky, new and better ways to achieve our nation's goals in space need to be addressed. Small, low cost spacecraft that can be developed in less than 3 years are being considered as the wave of the future in space science.

Many of these small spacecraft will have missions in and around Earth orbit. The low Earth orbit (LEO) is characterized by constant and frequent eclipses of the sun requiring high quality and long life batteries. Power requirements in the $1 \mathrm{~kW}-5 \mathrm{~kW}$ range will be typical. Solar arrays and flight rated batteries will be the typical source of electrical energy.

These similarities in the power system requirements can lead directly to similar power systems. However, it is still common for a new spacecraft to completely develop a power system from the ground up. The cost of custom made space electronics is extremely high and accounts for a major portion of the spacecraft power system development cost. Today's commercial electronic parts are extremely reliable and can possibly be used in spacecraft power systems to reduce costs. Also, by modularizing key power system components, much time, manpower, and money can be saved by allowing spacecraft designers to "build" their power system with offthe-shelf components designed to work together. This building block approach results in inexpensive spacecraft and short development times.

NASA Lewis Research Center has established the necessary groundwork and expertise in power systems and controls to investigate advanced modular electric power system (EPS) architectural concepts for small spacecraft. A small test bed based on a modular power system concept has been developed using the Electric Power System Test Bed in the Power Systems Facility at NASA Lewis. This world-class test facility was developed in support of the independent evaluation of the Space Station power system design. 1 


\section{EPS DESIGN FACTORS}

In a photovoltaic power system, several unique characteristics of the energy source and storage systems must be accounted for in the system design. These characteristics usually result in system requirements which address problem areas and make the most efficient use of the energy generated. Some examples are requirements for a fault tolerant power system and use of a "utility-type" power distribution. These requirements will greatly affect both the component and overall system design.

\section{Photovoltaic Arravs}

Since the 1960's solar cell arrays have been the choice for power generation in space. Their low mass, relatively long life, and ease of construction make them attractive electrical power sources. However, disadvantages include a low efficiency (typically below $17 \%$ ), large surface area, complex deployment mechanisms, and degradation over time.

Solar arrays have unique power generation characteristics which present challenges to the EPS design. They are characterized by a well known current vs. voltage (I-V) curve which is directly related to the solar cells' diode-like construction. Figure 1 shows a typical I-V curve and its corresponding P-V curve (power vs. voltage). The $I-V$ curve is defined by -

Isc - the short circuit current

$V_{O C}$ - open circuit voltage

$V_{m p}$ - maximum power point voltage

These characteristics are largely determined in the construction of the array by wiring the individual solar cells in series and parallel combinations. However, these cell characteristics can vary over time and affect the power generation capability of the array.

Short circuit current is dependent on the insolation which strikes the cells. This can be reduced due to degradation of the protective covering, contamination, or by simply moving farther away from the sun. The solar array open circuit voltage is a function of array temperature. Cold arrays emerging from eclipse have a period of high $V_{\text {oc }}$ until the array heats up to its nominal temperature. The maximum power point is a function of all the above-it moves with both Isc and $V_{O C}$.

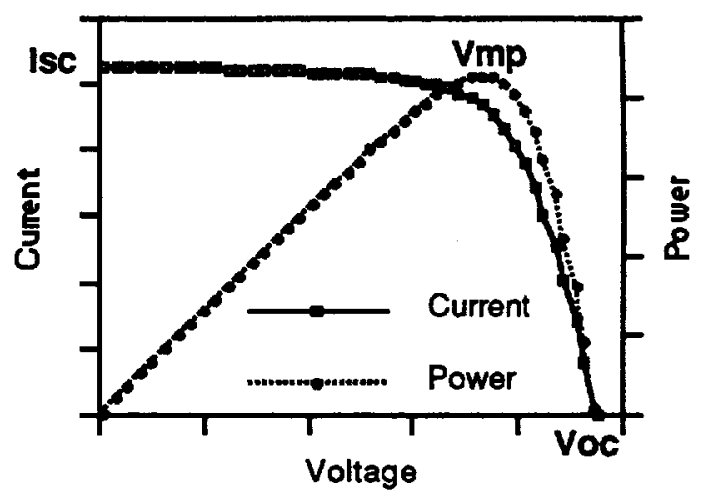

Figure 1 - Solar Array I-V Curve

In sizing solar arrays for spacecraft missions the EPS designer must take these factors into account. The array must be sized so that its end-of-life (EOL) power is sufficient to meet spacecraft power demands. Components must also be able to handle the high voltages generated as cold arrays emerge from eclipse.

\section{Battery Charoe Management}

Battery technology has been challenged by modern spacecraft which are smaller, lighter, and required to operate over longer periods of time. Especially challenging is the operation in low Earth orbit (LEO) which is characterized by frequent charge-discharge cycles and long eclipse times of up to $30 \%$ of the orbit. By increasing depth of discharge (DOD), the battery size and weight can be reduced while still providing the energy storage needed. However, increased DOD reduces the battery operating life. This poses problems to EPS designers who are forced to balance battery DOD with long operating lives.

Battery lifetime and energy density can be maximized using established charge management guidelines. Controlling the charge current during insolation can have a great impact on battery life span and size. Charge management can prevent overheating of the cells and extend the lifetime of the battery. Also, the use of taper and trickle charge cycles can extend battery lifetimes. It's clear that careful charge management is necessary to optimize use of spacecraft batteries.

\section{Eault Tolerance}

Past charge management designs have relied on power converters between an unregulated solar 
array bus and the battery. These designs work well and can be efficient, but, the entire battery can be lost if the power converter fails.

The recent failure of the NOAA-13 meteorological satellite was attributed to a short circuit in the battery charger electronics which prevented the solar arrays from charging the batteries. A fault tolerant power topology would have isolated the fault and allowed the arrays to directly charge the batteries. Losing the battery charge management would have reduced the operating life of the spacecraft. However, the solar arrays could have charged the batteries and operated normally for some time.

Other designs have used bidirectional DC-DC converters to charge the batteries during insolation and to provide regulated voltage during eclipse. These converters tend to be large, inefficient, and subject to a single fault which will fail the energy storage system of the entire power channel.

Yet other methods have controlled the solar array output through highly efficient converters. The output of the array is varied to affect the charge current into the battery. Similar to the methods above, failure of the array regulator will disable the entire power channel.

\section{User Power Requlation}

In some power systems, the users' (experiments, instruments, and spacecraft loads) are connected directly to the battery bus. In most cases these loads require DC-DC converters to convert the unregulated bus to usable voltage levels (typically 5 and $\pm 12 \mathrm{Vdc}$ ). These load converters must handle large voltage swings since the battery bus voltage can vary by up to $25 \%$ through the full range of charge and discharge cycles. These converters tend to be larger and less efficient than a converter which is designed for a specific input voltage level.

A "utility-type" power system delivers power to the users at a tightly regulated voltage. To accomplish this, the spacecraft EPS must provide regulation during insolation and eclipse periods, usually by means of a DC-DC converter. The converter is the interface between the primary, or source, bus and the secondary bus. By providing a regulated voltage to the user loads their DC-DC converter designs can be optimized for a specific input voltage. The smaller, more efficient converters will have a positive impact on the overall spacecraft size and weight.

\section{SERIES CONNECTED BOOST CONVERTER}

A new power converter interconnection technology has been developed at the NASA Lewis Research Center which is ideally suited for photovoltaic power systems. The converter is called a series connected boost converter (SCBC) and provides size, weight, efficiency, and fault tolerance benefits over existing technologies. 2

The SCBC is based on an isolated, step-down DC-DC converter. A block diagram of the SCBC is shown in Figure 2. Because the converters' output is isolated from the input it can be connected such that the output is in series with the input. By referencing the output voltage to the input return line, the resulting output voltage is "boosted" from the input voltage by the converter. However, the converter only processes a fraction of the total power delivered. The amount is determined by the ratio of boost voltage to output voltage. The majority of the current simply bypasses the DC-DC converter, passing through the transformer secondary windings and the rectifier diodes.

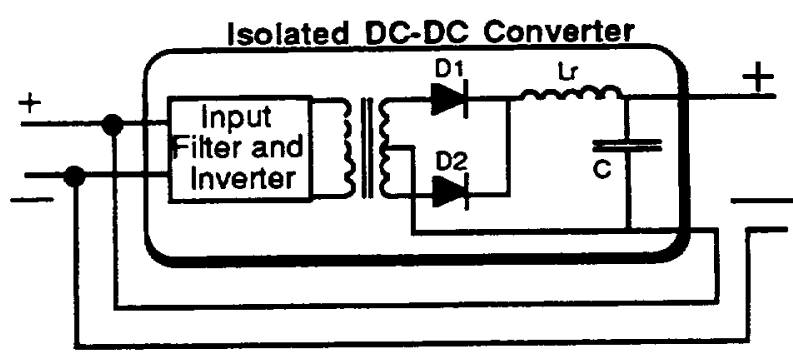

Figure 2 - Series Connected Boost Converter

\section{Power Density and Efficiency}

The series connected boost connection allows a converter to control more than its rated power level. In the SCBC, the DC-DC converter processes only a fraction of the total power delivered; most of the power is bypassed through the series connection. Consequently, the size and weight of the SCBCs are smaller than a similarly rated, conventional DC-DC converter.

The SCBC is very efficient since it only has to process a portion of the total power delivered. Any power that bypasses the converter 
experiences only filter losses and diode conduction losses. Efficiencies in the lab have been measured between $95 \%$ and $98 \%$ using standard, isolating DC-DC converters which had individual efficiencies of $85 \%$ to $90 \%$. An illustration of how the SCBC leverages power and improves efficiency is given below:

DC-DC Converter Specs. (225W)

Input $=21-56 \mathrm{Vdc}$

Output=15 Vdc @ 225W

Efficlency $=90 \%$

800 W SCBC (zero boost)

Input $=55 \mathrm{Vdc}$

Output = 55 Vdc @ 14.5 A

Diode losses $=14.5 \mathrm{~A} \cdot 0.7 \mathrm{~V}=10 \mathrm{~W}$

Filter losses $=5 \mathrm{~W}$

TOTAL Losses $=15 \mathrm{~W}=\mathbf{9 8 . 1 3} \%$ efficient.

800 W SCBC (15Vdc boost)

Input $=40 \mathrm{Vdc}$

Output $=55 \mathrm{Vdc} \cdot 14.5 \mathrm{~A}$

Converter $=15 \mathrm{Vdc} \cdot 14.5 \mathrm{~A}=218 \mathrm{~W}$

$90 \%$ efficient $=22 \mathrm{~W}$ of converter losses

Add fixed losses $=15 \mathrm{~W}$

TOTAL losses $=37 \mathrm{~W}=\mathbf{9 5 . 3 8} \%$ efficlent.

Obviously the gains in efficiency depend upon the DC-DC converter efficiency, the amount of fixed losses, and the boost voltage to output voltage ratio.

\section{Fault Tolerance}

Perhaps the most significant advantage of the series connected boost converter over other power converters is its ability to mitigate a complete failure of the power electronics. In other converters, such as the buck regulator, a failure of the power electronics or control circuits will completely disable the power delivery of that converter. However, the SCBC's unique connection allows for power to bypass a failed converter through the transformer secondary windings and rectifier diodes. Even when the DC-DC converter in the SCBC fails, the "zero boost" power is still available at the output of the converter. This fault tolerance characteristic makes the SCBC an ideal interface between a solar array and a battery.

\section{Solar Arrav to Battery Interface}

The SCBC can be used to regulate the battery charge current by controlling its boost voltage. Even as the array characteristics change (Isc and $\left.V_{O C}\right)$, the SCBC is able to control battery charge current while delivering full power to the system. However, it is important that the array and batteries be sized properly for the SCBC operation at the extreme conditions. At the beginning of life (BOL) the cold array will produce excess power. However, the battery charge current cannot exceed Isc and the voltage will be clamped to the battery. At the end of life (EOL) the degraded array must operate at the peak power point to deliver sufficient power. The SCBC must be sized so that the array voltage at maximum power, $V_{m p}$, can be boosted to provide full battery charge current.

\section{Commonality}

Finally, the series connected boost converter makes an excellent "building block" for a photovoltaic power system. By using a voltage regulator circuit the SCBC can be used to deliver regulated battery voltage to the spacecraft loads. Similarly, a SCBC can control battery charge current by simply adding a current regulator circuit. The DC-DC converter can also be used in its native mode to isolate a sensitive load from the spacecraft power system. By using one common DC-DC converter as a building block for the entire electric power system both, development costs and flight hardware costs can be reduced.

\section{TROPIX EPS TEST BED}

The recent failure of the ANIK 2 satellite due to transient, high energy fields in the Earth's magnetosphere have led to a proposed science satellite named TROPIX (Transfer Orbit Plasma Interaction Experiment). 3 The main purpose of the TROPIX satellite is to fully map the Earth's magnetosphere and investigate-

Magnetic and Electric Fields,

Low energy plasma, and

Energetic particles.

By fully understanding the Earth's magnetosphere, spacecraft can be designed to withstand the high energy plasma fields and mitigate damage to the electric power system.

A secondary mission of the TROPIX spacecraft is to demonstrate the feasibility of solar electric propulsion. Low thrust solar electric propulsion (SEP) allows a slow, nearly circular orbit procession from low Earth orbit to beyond geosyncronous orbits. Accuracy of the magnetic and electric field measurements improve as the TROPIX spacecraft 


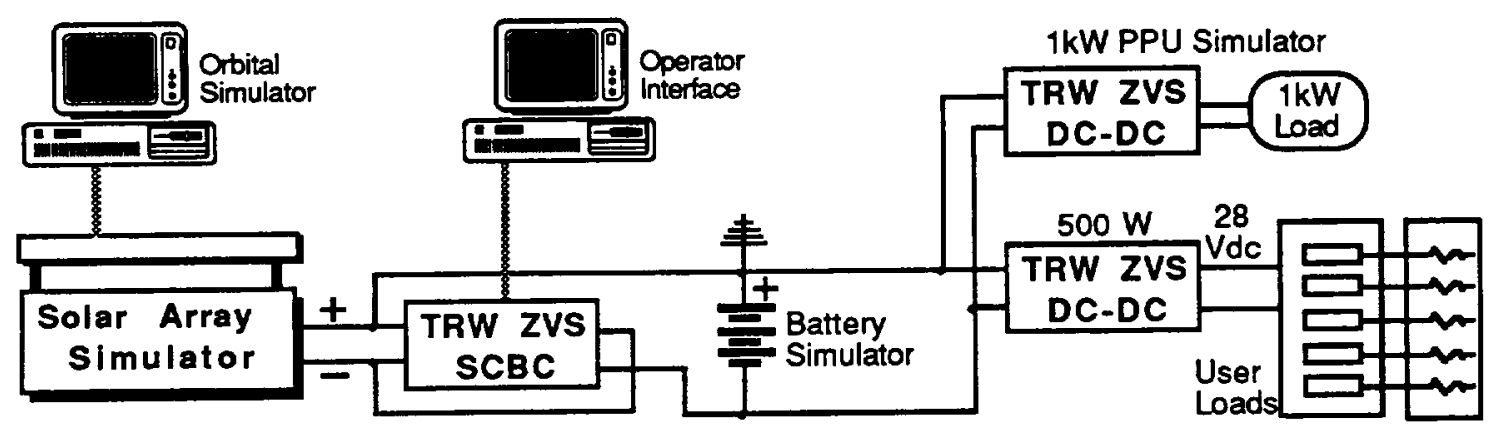

Figure 3 - TROPIX EPS Test Bed Block Diagram

slowly spirals through the magnetosphere.

The use of SEP for the TROPIX satellite places high importance on the electric power system. Power requirements include two (2) 900 Watt SEP engines and about 350 Watts of user loads (communications, navigation, and the science experiments) for a total power of $2.2 \mathrm{~kW}$. The TROPIX satellite must also have a positive-ground power system due to the nature of the plasma science instruments. By positively grounding the solar arrays, interaction with plasma fields are greatly reduced.

An electric power system test bed was developed for the TROPIX program as a technology demonstrator of a positive-ground power system based on the SCBC.

\section{Qbjectives}

Objectives of the TROPIX EPS Test Bed were to provide an inexpensive test capability for the program and to demonstrate the power system technology necessary for solar electric propulsion. By providing a system test capability, the series connected boost converter topology in a positive ground configuration could be developed and evaluated. System level tests will provide data for power system sizing and control. In addition, data acquisition requirements could be defined. This Phase I test bed development effort was completed in seven months.

\section{Test Bed Description}

The TROPIX EPS Test Bed block diagram is shown in Figure 3. To reduce development costs, the test bed was constructed using power supplies, bulk DC-DC converters, and a solar array simulator used in the Space Station independent verification program at the NASA
Lewis Research Center. The hardware was modified to meet the functional requirements of the TROPIX spacecraft. However, the component ratings and sizes were larger than required. Also, this Phase I test bed simulated only one of the two SEP power channels.

The TROPIX test bed is a positive-ground design based on the series connected boost converter. The SCBC is used as the interface between the solar array simulator and the battery. A current regulator was added to the SCBC so the battery charge current would be controlled during insolation. The system is loaded using two large DC-DC converters ( $1 \mathrm{~kW}$ each).

A control and data acquisition system developed for the Space Station test bed program was modified for use in the TROPIX EPS Test Bed. The complex, distributed, software control system developed for the Space Station test program was greatly simplified and implemented on a single desktop computer. Through the Operator Interface the test engineer can: turn the SCBC on and off, set current and voltage setpoints, and monitor the output voltage and battery current.

An orbital simulator was also developed for the TROPIX test bed. The orbital simulator accurately simulates any circular orbit using the facility control system and the solar array simulator. By entering several orbital parameters, the orbital simulator: calculates the orbit period, calculates max., min., and nominal eclipse times, and simulates the array heating following eclipse. The orbital simulator is an invaluable tool in evaluating power system performance through several orbits.

\section{Series Connected Boost Converter}

The SCBC used in the TROPIX EPS Test Bed is built around a $1 \mathrm{~kW}$ zero voltage switching DC-DC 
converter built by TRW of Redondo Beach, CA. It was designed to convert $120 \mathrm{Vdc}$ to an isolated 28 Vdc for user loads on the Space Station ${ }^{4}$. The converter was modified to a positive ground, series connected boost converter to provide an output voltage range from -90 to $-117 \mathrm{Vdc}$.

Initial development of the SCBC focused on providing output voltage regulation in a positive ground configuration. Since the voltage regulator is optically isolated from the power circuit, the positive ground configuration was trivial. The resulting SCBC had an output power capability of about $4.25 \mathrm{~kW}$ at an input voltage of $100 \mathrm{Vdc}$ and an output voltage of $117 \mathrm{Vdc}$. The efficiency of the SCBC was measured using two calibrated Norma watt meters. The results are shown in Figure 4. The efficiency ranged from $95 \%$ to $98 \%$ and had an average efficiency of $96.4 \%$. The efficiency numbers also include the control power since it is generated internal to the TRW ZVS converter.

Since the SCBC needed to control the charge current into the battery, a current control circuit was integrated with the existing voltage control circuit. The battery current is sensed (using a Hall effect sensor) and is compared to a reference. The current regulator adjusts the SCBC's boost voltage to control battery charge current. However, if the battery voltage reaches a pre-set maximum (defined by the voltage regulation setpoint) the converter reverts to a voltage regulation mode to protect the batteries from overcharging. The response of the current control circuit is $1 \mathrm{kHz}$ and is much slower than the voltage control circuit.

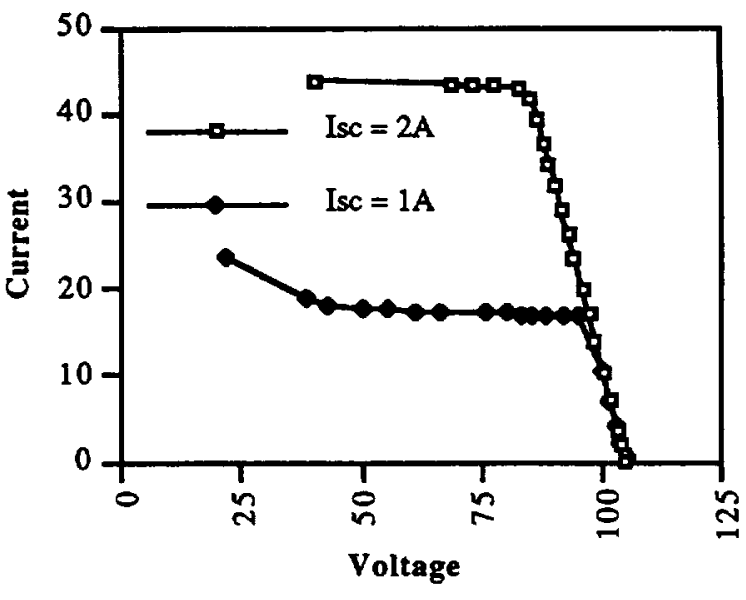

Figure 5 - Solar Array Simulator I-V Curve

\section{Solar Array Simulator}

The solar array simulator (SAS) was developed at Lewis to support testing of Space Station Freedom breadboard hardware and end-to-end system tests. It is an $\mathbf{8 2}$ string simulator which uses a large DC power supply and 82 linear power circuits to simulate the solar array I-V curve $^{5}$. For the TROPIX EPS Test Bed, 24 strings of the simulator were re-wired for a positive ground configuration. The 24 circuits have a common positive bus and supply 24 separate refurn circuits. An adjustable short circuit current knob can reduce the power delivered by the SAS. The SAS I-V curve is shown in Figure 5 with the power supply, or Voc, set to $105 \mathrm{Vdc}$ and Isc set to $2 A$ and $1 A$ per string. Although the "knee" of the simulator is more pronounced than that of an actual array (see Figure 1), the SAS provides a realistic simulation for testing.

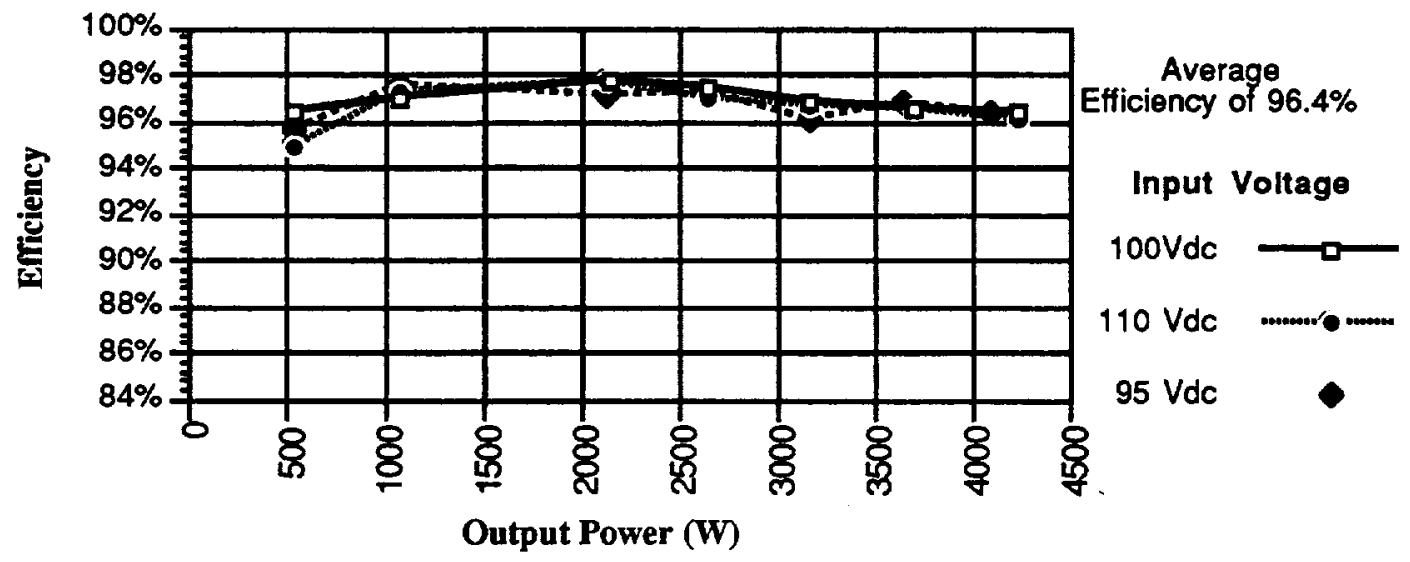

Figure 4 - Series Connected Boost Converter Efficiency Measurements 


\section{Batten Simulator}

The battery simulator consists of eight (8) sealed, lead-acid batteries. Connected in series, they provide 90-117 Vdc and have a capacity of $80 \mathrm{~A}-\mathrm{hr}$. This battery simulator is not the appropriate size battery expected for the TROPIX satellite but was used since it was readily available.

\section{Electrical Loads}

The TROPIX Test Bed includes simulated user loads of the size and type expected on the spacecraft. By using realistic loads, not just laboratory resistive load banks, the system can be accurately evaluated for power quality and transient response. The same 1kW TRW ZVS isolating converter used for the SCBC is used as a bulk load converter. It converts the high voltage battery bus to $28 \mathrm{Vdc}$ and isolates the loads from the positive ground. Connected to the output are several low power DC-DC converters of various sizes. Together they dissipate about $500 \mathrm{~W}$ through large power resistors mounted on a cold plate.

Another 1kW TRW ZVS isolating converter is used to simulate the response of one of the $900 \mathrm{~W}$ solar electric propulsion engines. The converter is loaded by a programmable load bank. The constant power DC-DC converter closely simulates the large signal dynamics of an SEP power processing unit (PPU). There are plans to incorporate an SEP PPU into the test bed in 1995 for complete system characterization.

\section{System Test Results}

The TROPIX EPS Test Bed has recently completed end-to-end integration and is currently undergoing system level tests. System tests planned include: power quality, efficiency, load transients, system faults, and orbital transition tests.

Results from the battery charge current regulator tests are shown in Figure 6 . This test observed the battery voltage, battery charge current, and solar array simulator voltage over an 8.5 minute period as the SCBC charged the battery at a programmed level of 10A. During the charge period the system loading was varied several times to verify correct current regulation. Initial loading was $500 \mathrm{~W}$ and the battery voltage was $90 \mathrm{Vdc}$. For one minute the $500 \mathrm{~W}$ load was supplied by the battery and the charge current is $-6.35 \mathrm{~A}$ ( $570 \mathrm{~W}$ delivered to the PPU simulator). At about one minute power was applied to the solar array simulator and the SCBC began to charge the battery at the $10 \mathrm{~A}$ setpoint level. During the next four (4) minutes the PPU load was varied from $900 \mathrm{~W}$ to $200 \mathrm{~W}$ while the battery charge current remained a steady $10 \mathrm{~A}$. At about five (5) minutes the battery voltage had reached the maximum voltage setpoint of $116.7 \mathrm{Vdc}$ and the battery current slowly tapered off as the voltage regulator gained control of the converter. During this time the PPU simulator load was still being varied but did not have an affect on the battery voltage or charge current.

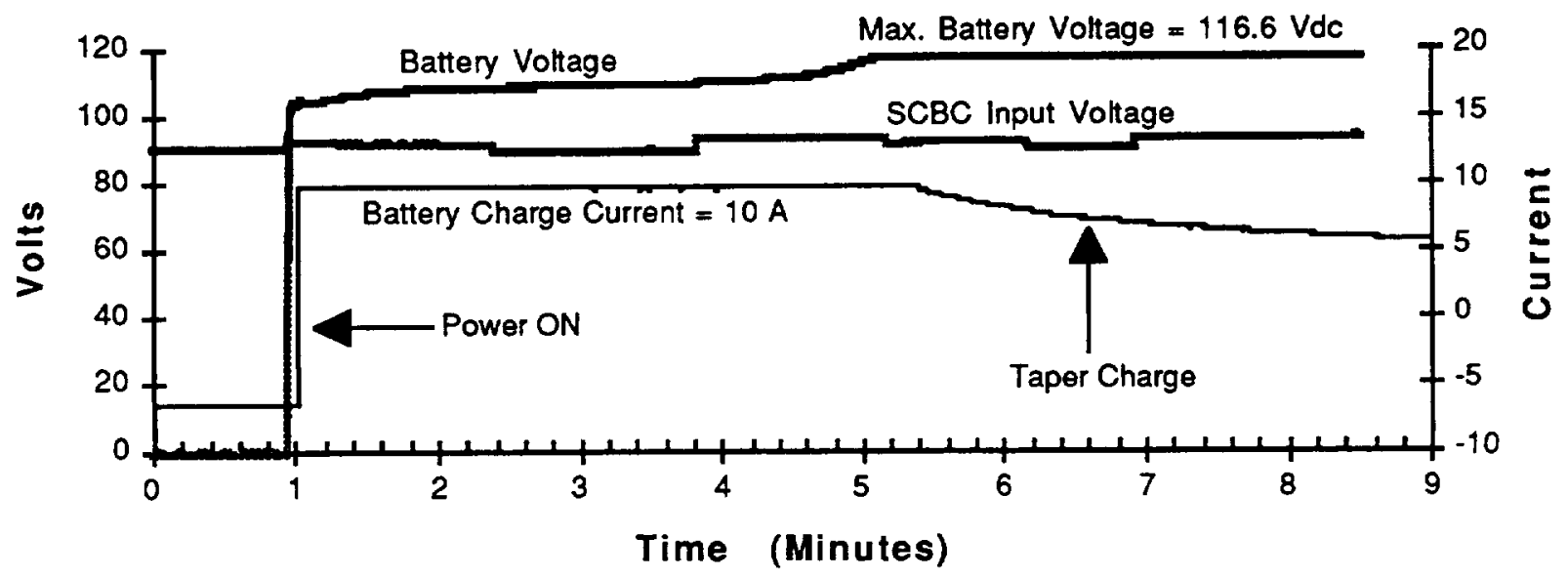

Figure 6 - Battery Charge Current Regulator Performance 


\section{PHOTOVOLTAIC REGULATOR KIT}

Using the experience gained in the TROPIX EPS Test Bed, a concept has been developed which will improve small spacecraft power systems while also reducing development time and program costs. The concept is based on the series connected boost converter (SCBC) and involves development of a pre-engineered "kit" for fast and inexpensive development of an electric power system. This PV regulator kit is modular and can be easily paralleled to provide a range of power levels for small spacecraft power systems.

\section{Description}

A block diagram of the PV regulator kit concept is shown in Figure 7 . The kit includes: a small housekeeping power supply, communications interface, input and output filters for improved power quality, a battery current monitor for charge control, and a bus voltage regulator and paralleling interface. The DC-DC converters used as the SCBCs can be any type of isolating power supply with an external interface for controlling output voltage. By not defining a specific DC-DC converter the kit can be used with almost any converter, including off-the-shelf commercial parts. This provides the EPS designer flexibility in the size of the power channel. Also, since DC-DC converters are used to power other spacecraft systems and experiment, a common converter can be used, further reducing EPS development costs.

\section{Operation}

The PV regulator is the interface between a properly sized solar array, a battery, and a secondary power bus. The first SCBC is controlled by the battery charge control circuit. By varying the SCBC boost voltage, the battery charge current can be controlled to follow a predefined profile. This profile can be controlled from an external computer or can be embedded in firmware in the communications interface. Should the DC-DC converter fail, current from the solar array will still charge the batteries.

The second SCBC is the interface between the battery bus and the secondary distribution bus. This converter supplies regulated voltage to the user loads which reduces their power converter complexity. This SCBC is controlled by the bus regulation controller which monitors the secondary bus voltage and adjusts the SCBC to keep the output voltage at a regulated setpoint value. The input and output filters are necessary to improve power quality and to isolate current and voltage ripple noise which might otherwise be passed directly to the user loads.

Not depicted in the block diagram is the fault protection system which consists of strategically placed fuses. Each SCBC has a fused input so that a fault in either DC-DC converter will not clamp the energy sources. Because the solar array is a current limited source, no fuse is needed to protect the wires from high fault currents. However, the battery can easily create large fault currents so the battery will be fused to protect against short circuits in the series connected boost wiring.

\section{Modularity}

The PV regulator kit is designed to be modularallowing several units to be paralleled for greater

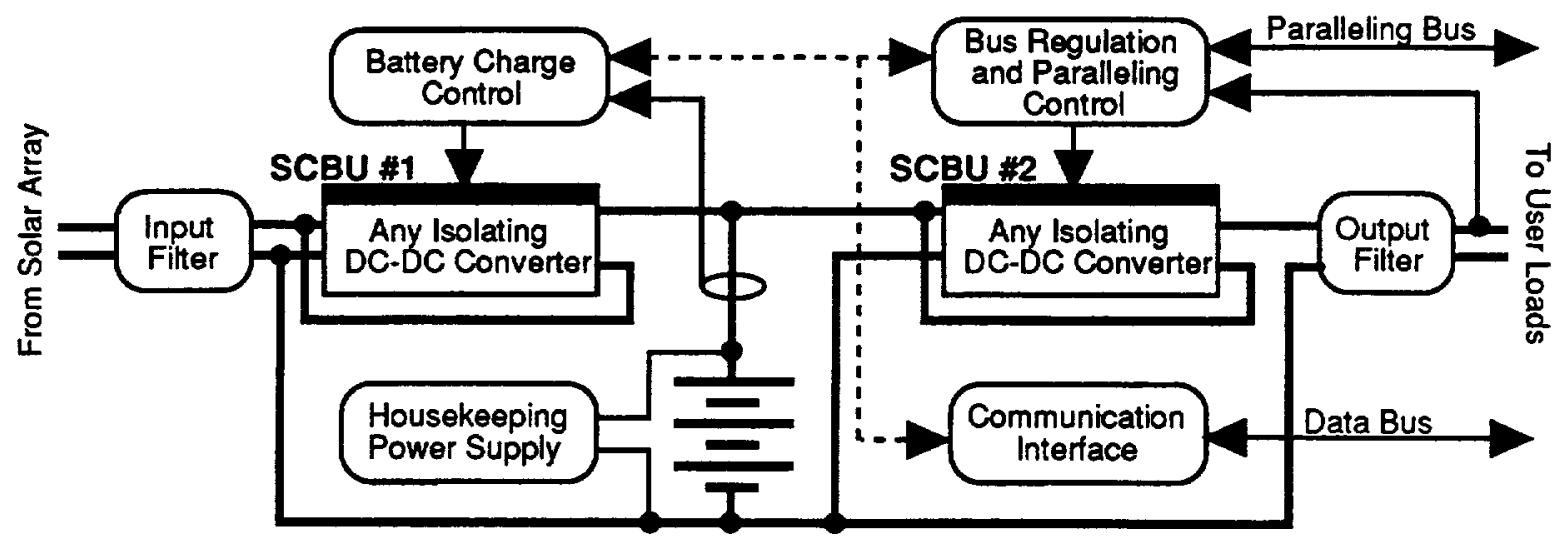

Figure 7 - Photovoltaic Regulator Kit Block Diagram 
power delivery to the user loads. The paralleling is controlled through the regulated output bus and is an integral part of the bus regulation controller. Paralleling is accomplished through a shared signal bus which forces the converters to equalize their output currents with other units connected to the bus.

\section{Elight Experiment}

A version of the PV regulator kit is being developed for flight testing in the Small Spacecraft Technology Initiative (SSTI) program sponsored by NASA. Scheduled to fly in 1996, the SSTI satellite will test new technologies that can greatly impact the future costs and complexities of small spacecraft.

\section{CONCLUSIONS}

Based on the unique requirements of photovoltaic power systems, an optimal design can be achieved using the series connected boost converter (SCBC). The SCBC provides size, weight, power density, efficiency, fault tolerance, and commonality benefits over existing technologies. Also, electric power system development time and costs can be reduced by using commercial DC-DC converters as the SCBCs.

Development of the TROPIX EPS Test Bed has proven some of the benefits of the SCBC. Converter efficiencies of $95 \%$ to $98 \%$ have been measured in the lab. Use of the SCBC in a positive ground power system was also demonstrated. Further refinement of the test bed will focus on development of a Photovoltaic Regulator Kit. This pre-engineered power system element is modular and benefits from the SCBC technology. Future power systems can be "buitt" using off-the-shelf hardware-resulting in smaller, faster, and cheaper spacecraft.

\section{ACKNOWLEDGMENTS}

The author would like to acknowledge the work of Raymond Beach and Andy Brush in the development of the series connected boost converter.

\section{REFERENCES}

1 L. Trase, D. Fong, V. Adkins, A. Birchenough, "Description of the PMAD Systems Test Bed Facility and Data System", 27th Intersociety Energy Conversion Engineering Conference, San Diego, CA, August 1992.

2 R.F. Beach, A.S. Brush, "Series Connected Converter for Control of Multi-Bus Spacecraft Power Utility", NASA Disclosure of Invention, \#LEW15918-1C, Nov 1993.

3 J. Mark Hickman, "Solar Electric Propulsion for Magnetospheric Mapping", 30th

AIAAJASME/SAE/ASEE Joint Propulsion Conference, June 27-29, 1994.

4 R. Lebron, "Load Converter interactions with the Secondary System in the Space Station Freedom PMAD DC Test Bed", 27th Intersociety Energy Conversion Engineering Conference, San Diego, CA, August 1992.

5 T. Vasek, A. Birchenough, "Development of a Ninety String Solar Array Simulator", 26th Intersociety Energy Conversion Engineering Conference, Boston, MA, August 1991. 


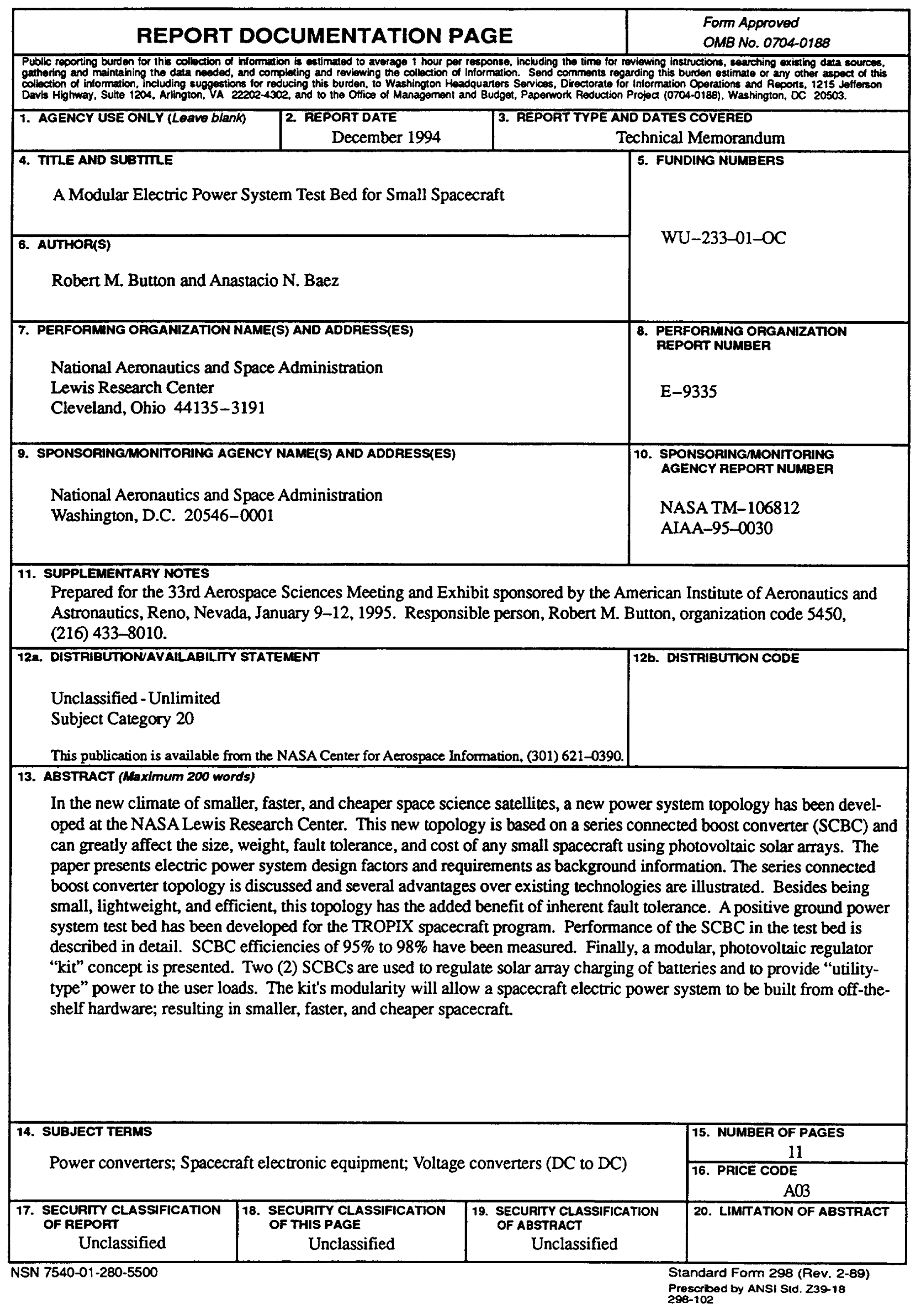




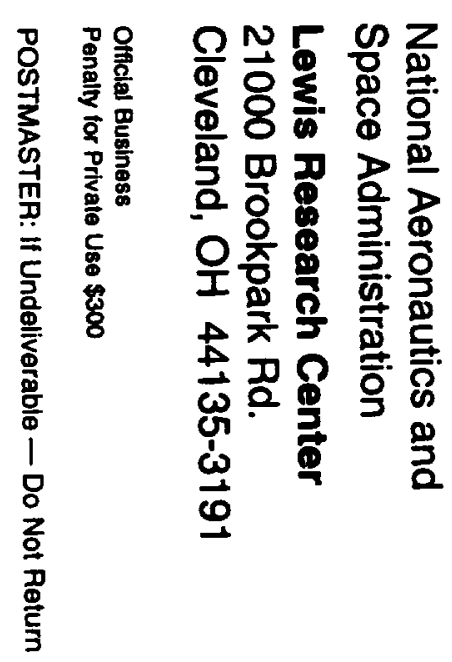


